\title{
Performance of five sweet orange (Citrus sinensis) cultivars on four rootstocks under Maharashtra State, India
}

\author{
Dhake Anil Vasudeo ${ }^{1}$, Patil Dnyaneshwar Gambhirrao ${ }^{1} \&$ Balkrishna $^{1}$
}

\section{SUMMARY}

Evaluation of five sweet orange cultivars, viz. Hamlin, Valencia, Pera, Westin and Natal on four rootstocks, viz. Volkameriana, Swingle citrumelo, Cleopatra tangerine and Rangpur lime was conducted during 2014 and 2015 on four year old plantation in central India (Jalgaon) under sandy loam soil. Maximum plant eight was found in Hamlin on Volkameriana produced while Pera was dwarfest on Cleopatra tangerine as compared to other rootstock scion combinations. Rootstock average value tallest to dwarf plants were Volkameriana followed by Rangpur lime, Swingle citrumelo and Cleopatra tangerine however for scion varieties this order was Hamlin followed by Valencia, Natal, Westin and Pera. Hamlin on Swingle citrumelo produced largest canopy and Pera on Cleopatra tangerine produced smallest canopy volume. Among the rootstocks Swingle citrumelo produced largest and Cleopatra tangerine produced smallest canopy. The maximum scion circumference was observed in Hamlin and minimum in Westin. Valencia recorded maximum value for fruit weight. Maximum juice content was observed in Pera while highest TSS level was observed in Hamlin. Natal followed by Hamlin and Valencia budded on Swingle citrumelo registered higher yield (67.10, 62.6, 59.5 kg plant $\left.{ }^{-1}\right)$ during 5th year of plantation however lowest yield was observed in Valencia budded on Volkameriana. We could categorize yield in four group very good $(>50)$, good (35-50), average (20-35) and poor $(<20)$. Hamlin, Natal and Valencia on Swingle citrumelo can be grouped into very good yielder, Hamlin, Natal, Valencia on Cleopatra tangerine, Natal and Westin on Rangpur lime and Westin on Volkameriana can be categoral as good yield. Rest of the combinations was in average yielding category except value on Volkameriana was a poor yielder.

Index terms: sweet orange, rootstocks, cultivars, yield.

\section{Desempenho de cinco cultivares de laranja doce (Citrus sinensis) em quatro porta-enxertos no Estado de Maharashtra, Índia}

\section{RESUMO}

Avaliações de cinco cultivares de laranja doce: Hamlin, Valência, Pera, Westin e Natal em quatro porta-enxertos: limão Volkameriano, citrumelo Swingle, tangerina Cleopatra e limão Cravo, foram conduzidas durante 2014 e 2015 em plantação de quatro anos no centro da Índia (Jalgaon) sob solo arenoso. A planta mais alta foi a Hamlin enxertada em Volkameriano, enquanto a Pêra foi a que menos cresceu quando enxertada na tangerina de Cleópatra em comparação com outras

\footnotetext{
${ }^{1}$ Jain R \& D, Agri Park, Jain Irrigation Systems Ltd., Jalgaon State, Maharashtra, India

Corresponding author: Dhake Anil Vasudeo, Jain R \& D, Agri Park, Jain Irrigation Systems Ltd., NH No. 6, Bambhori, Jalgaon (425 001) Maharashtra, India. E-mail: dr.dhake.anil@jains.com
} 
combinações. Dentre os porta-enxertos as plantas ficaram menores no Volkameriano seguido de limão Cravo, citrumelo Swingle e tangerina Cleópatra, no entanto, para variedades copa, a ordem foi Hamlin seguida de Valência, Natal, Westin e Pera. Hamlin em citrumelo Swingle produziu o maior volume de copa e Pera em Cleopatra tangerina produziu o menor. Entre os porta-enxertos citrumelo Swingle produziu maior e a tangerina Cleopatra produziu o menor volume de copa. A circunferência máxima do tronco foi observada em Hamlin e mínima em Westin. Valência registrou valor máximo para o massa do fruto. O teor máximo de suco foi observado em Pera, enquanto o teor de sólidos solúvies mais alto foi observado em Hamlin. Natal seguido por Hamlin e Valência enxertadas em citrumelo Swingle registaram maior produtividade $\left(67,10,62,6,59,5 \mathrm{~kg} \mathrm{planta}^{-1}\right)$ durante o $5^{\circ}$ ano de plantação, porém o menor rendimento foi observado em Valência enxertada em Volkameriano. Podemos agrupar a produtividade em quatro grupos: muito bons $(>50)$, bons (35-50), médios (20-35) e ruins $(<20)$. Hamlin, Natal e Valência em citrumelo Swingle podem ser agrupados em muito bons, Hamlin, Natal, Valência em Cleopatra; Natal e Westin em Cravo e Westin em Volkameriano podem ser agrupados como os de boa produtividade. As demais combinações ficaram na categoria de rendimento médio, exceto o Volkameriano, que foi o pior em produtividade.

Termos de indexação: laranja doce, porta-enxertos, cultivares, rendimento.

\section{INTRODUCTION}

Citrus is one of the leading economically important fruit crops grown in more than 50 countries under tropical and sub-tropical region of the world with 7.48 million hectares area and 110.0 million metric ton production. India grows citrus fruits on 1.08 million hectares with production of 11.15 million metric tons and having third rank accounting for about 14.9 and 12.5 per cent of the area and production of fruits, respectively (NHB, 2015).

India grows a range of citrus species like lime, lemon, sweet orange and mandarin. Among these, mandarin occupies largest area in Maharashtra (135 thousand ha) followed by Madhya Pradesh (52.5 thousand ha) and Punjab (47 thousand ha) but productivity in Maharashtra is very poor, only 5.5 MT/ha (NHB, 2015). Besides mandarin, Mosambi a very low acid sweet orange is grown in India on large scale with a total area of 0.334 million hectare and production of $3.43 \mathrm{~m} \mathrm{MT}$, Maharashtra grows Mosambi on 95 thousand hectare which about 18 percent of total production. Though this variety is well adapted to central India and produces well even under marginal conditions. This variety is primarily used for freshly squeezed juice and consumed with added sugar and salt or other spices and condiments. It is a low acid, low sugar and high Limonin content thus unfit for processing. There is need to have sweet orange variety which is high yielding with high TSS, good sugar acid ratio and low limonin content. It was attempted to introduce sweet orange varieties in India to desired fruit and juice quality.

Besides scion variety, rootstocks play an important role in citrus cultivation to success and failures in citrus processing industry. The strong effect of rootstock on tree growth yield and quality of fruiting has been investigated.
In addition to supporting the tree in soil, the rootstock responsible for absorption of water and nutrients, thus altering increase of tree canopy and photosynthesis, providing storage of carbohydrate, adaption tolerance of some diseases. The rootstock effect on tree growth, fruit quality and production has been widely elaborated and different parameters of fruit crops have been advocated by them.

Majority of citrus plantation in Maharashtra state is on Rangpur Lime rootstock. This stock has suitable characters like drought tolerance and extensive root system for high vigor, large tree, high yield and early production but susceptible to Phytophthora, which has resulted into lowering the productive age of orchards. In focus of important roles played by rootstocks and drawbacks of presently used stock in state, the opportunity need for new rootstock is major concern. It urgent need for focus of new varieties and rootstock in Maharashtra.

Keeping in view sweet orange plants of five varieties with four rootstock combinations were in imported from Brazil. The present study investigated the performance of these varieties in term of growth, yield and fruit quality characters to expand varietal diversity and better-quality juice.

\section{MATERIAL AND METHODS}

The experiment was carried out at Jain R \& D farm, Jalgaon, Maharashtra (India) during 2014 and 2015 (Crop age $4^{\text {th }}$ and $5^{\text {th }}$ year). The demonstration farm comes under the North Maharashtra and represents typical sub-tropical climatic condition (Latitude, $21^{\circ} \mathrm{N}$ and Longitude $75^{\circ} \mathrm{E}$ ) the daily maximum temperature is 
$42.7^{\circ} \mathrm{C}$ and the mean minimum temperature is $12.1^{\circ} \mathrm{C}$ with annual average rainfall of $749 \mathrm{~mm}$.

Five sweet oranges varieties, Hamlin, Natal, Pera, Valencia and Westin were planted on high density plantation at Jain R \& D farm, Jalgaon in 2010. There were imported as finished plants budded on different rootstocks: Swingle citrumelo (CS), Cleopatra tangerine (TC), Volkameriana (Vol) and Rangpur Lime (RL) from Brazil. Plantation was done with 4 meter row to row and 3 meter plant to plant spacing.

The different stock-scion combinations were studies for the performance in term of vegetative growth characters, yield and fruit quality characters. The data for seven parameters was recorded during 2014 and 2015. PVC pole, calibrated in feet and inches was used to measure the height of each sample plant. Height was measured from bud union to the top of plant. Tree size was derived in term of Canopy Volume (CV) with the help of calculation, $\mathrm{CV}=0.542 \mathrm{hd}^{2}$ which is one-half of a prolate spheroid with $\mathrm{h}$ denoting as tree height, $\mathrm{d}$ donate average of N-S and E-W diameters. Scion circumference was measured with the help of measuring tape, just above and below the bud union. Yield was computed from the average of sample plants.

Random samples of 20 fruits of same colour and almost the uniform size were harvested at optimum maturity and immediately transported to Biochemistry lab of Jain R \& D, Jalgaon for Physico-chemical analysis. Fruit weight was recorded with the help of electronic balance. Juice content was extracted using citrus juice extractor and expressed digital hand refractometer (Atago, Japan). Fruit yield per plant was determined by harvesting and weighing the fruits from the center 10 trees of plot. Data were analyzed statistically by RBD design using statistical analysis systems software (Online software analysis) at 5 percent level of probability.

\section{RESULT AND DISCUSSION}

\section{Plant height}

Among five sweet orange varieties budded on four different rootstocks, plants of Hamlin variety produced maximum height in both years (Table 1). It was statistically at par with Valencia and Natal, which were the next to follow. Maximum plant height was found in Hamlin on Volkameriana while Pera was dwarfest on Cleopatra tangerine as compared to other rootstock and scion combinations. Rootstock average value tallest to dwarf plants were Volkameriana followed by Rangpur lime, Swingle citrumelo and Cleopatra tangerine however for scion varieties this order was Hamlin followed by Valencia, Natal, Westin and Pera. Similar result reported by Chahal \& Gill (2015) out of the total eighteen stionic combinations, Volkameriana rootstock contributed towards maximum plants height.

\section{Canopy volume}

The data show (Table 2) that Hamlin plants produced maximum canopy volume, followed by Valencia. Minimum canopy volume was recorded in Westin and Pera. The results regarding effect of rootstock on canopy volume revealed that plants budded on Swingle

Table 1. Plant height $(\mathrm{m})$ of five sweet orange cultivars on four rootstocks (Maharashtra State, India)

\begin{tabular}{|c|c|c|c|c|c|c|c|c|c|c|}
\hline \multirow{2}{*}{$\begin{array}{c}\text { Variety/ } \\
\text { Rootstock }\end{array}$} & \multicolumn{5}{|c|}{ Plant height $(\mathrm{m}) 4^{\text {th }}$ year } & \multicolumn{5}{|c|}{ Plant height $(\mathrm{m}) 5^{\text {th }}$ year } \\
\hline & $\mathrm{TC}$ & $\mathrm{RL}$ & $\mathrm{CS}$ & Vol & Varietal Mean & TC & RL & $\mathrm{CS}$ & Vol & Varietal Mean \\
\hline Hamlin & 2.6 & 2.9 & 2.8 & 3.0 & 2.81 & 3 & 2.9 & 2.9 & 3.0 & 2.95 \\
\hline Natal & 2.3 & 2.4 & 2.5 & 2.6 & 2.45 & 2.6 & 2.6 & 3.1 & 2.7 & 2.75 \\
\hline Pera & 2.2 & 2.3 & 2.3 & 2.5 & 2.31 & 2.5 & 2.4 & 2.8 & 2.5 & 2.52 \\
\hline Valencia & 2.6 & 2.8 & 2.6 & 2.7 & 2.65 & 2.7 & 2.8 & 3.0 & 3.0 & 2.85 \\
\hline Westin & 2.2 & 2.6 & 2.4 & 2.6 & 2.46 & 2.5 & 2.6 & 2.9 & 2.6 & 2.66 \\
\hline Interaction Mean & \multicolumn{4}{|c|}{$\begin{array}{l}\text { C.D. }-0.094 \text {, } \\
\text { SE(m)-0.033 }\end{array}$} & $\begin{array}{l}\text { C.D.-0.074, } \\
\text { SE(m)-0.066 }\end{array}$ & \multicolumn{4}{|c|}{$\begin{array}{l}\text { C.D. }-0.094 \text {, } \\
\text { SE(m)-0.033 }\end{array}$} & $\begin{array}{l}\text { C.D.-0.074, } \\
\text { SE(m)-0.026 }\end{array}$ \\
\hline \multirow[t]{2}{*}{ Rootstock Mean } & 2.4 & 2.6 & 2.5 & 2.7 & 2.54 & 2.6 & 2.7 & 2.9 & 2.8 & 2.74 \\
\hline & \multicolumn{5}{|c|}{ C.D. $-0.084, \mathrm{SE}(\mathrm{m})-0.029$} & \multicolumn{5}{|c|}{ C.D. $-0.066, \mathrm{SE}(\mathrm{m})-0.023$} \\
\hline
\end{tabular}

Cleopatra tangerine (TC); Rangpur Lime (RL); Swingle citrumelo (CS); Volkameriana (Vol); C.D. = coefficient desviation and SE - error standard. 
citrumelo registered maximum canopy growth followed by Volkameriana and Rangpur Lime. Cleopatra tangerine recorded minimum canopy volume. The canopy volume of different stoinic combinations studied showed that plants on Hamlin budded on Swingle citrumelo. Canopy volume for 7 year-old trees varied among cultivars, Hamlin $\left(6.6 \mathrm{~m}^{3}\right)$ and Valencia $\left(6.1 \mathrm{~m}^{3}\right)$ and rootstocks significantly affected tree size, Swingle and Sour orange are intermediate in vigor but they were among the largest in the experiment (Wheaton et al.,1991).

\section{Scion circumference}

Various sweet orange varieties did not significantly affect the scion circumference of the plant (Table 3). Maximum scion circumference was recorded in Hamlin, followed by
Valencia, while minimum value was recorded in Westin. Present investigation of sweet orange varieties observed variation in stem thickness among five varieties. Similarly scion circumference was not affected significantly by any rootstock used in study. Chahal \& Gill (2015) reported non significantly effect of rootstock on scion circumference of the plant in sweet orange.

\section{Fruit weight}

Two years data regarding influence of scion cultivar on fruit weight (Table 4) recorded that Natal produced heaviest fruits followed by Valencia and Westin. However, the variation between all these three varieties was statistically at par. Hamlin variety recorded by lowest fruit weight. However, fruit weight was not significantly

Table 2. Canopy volume $\left(\mathrm{m}^{3}\right)$ of five sweet orange cultivars on four rootstocks (Maharashtra State, India)

\begin{tabular}{|c|c|c|c|c|c|c|c|c|c|c|}
\hline \multirow{2}{*}{$\begin{array}{l}\text { Variety/ } \\
\text { Rootstock }\end{array}$} & \multicolumn{5}{|c|}{ Canopy volume $\left(\mathrm{m}^{3}\right)$ 4th year } & \multicolumn{5}{|c|}{ Canopy volume $\left(\mathrm{m}^{3}\right) 5$ th year } \\
\hline & $\mathrm{TC}$ & RL & $\mathrm{CS}$ & Vol & $\begin{array}{l}\text { Varietal } \\
\text { Mean }\end{array}$ & $\mathrm{TC}$ & RL & $\mathrm{CS}$ & Vol & $\begin{array}{l}\text { Varietal } \\
\text { Mean }\end{array}$ \\
\hline Hamlin & 9.9 & 11 & 12 & 12 & 11.2 & 9.9 & 11.3 & 13.8 & 12.20 & 11.78 \\
\hline Natal & 7.7 & 9.2 & 10.0 & 9.3 & 9.1 & 8.54 & 9.65 & 12.3 & 9.12 & 9.91 \\
\hline Pera & 5.4 & 6.0 & 6.8 & 9.0 & 6.8 & 6.18 & 7.29 & 10.3 & 9.07 & 8.21 \\
\hline Valencia & 11.0 & 11.0 & 9.8 & 8.5 & 10.1 & 13.1 & 10.7 & 12.5 & 8.93 & 11.29 \\
\hline Westin & 6.8 & 9.2 & 7.3 & 8.3 & 7.9 & 8.8 & 8.56 & 7.25 & 8.73 & 8.34 \\
\hline $\begin{array}{c}\text { Interaction } \\
\text { Mean }\end{array}$ & \multicolumn{4}{|c|}{$\begin{array}{l}\text { C.D. }-0.16 \text {, } \\
\text { SE(m)-0.056 }\end{array}$} & $\begin{array}{l}\text { C.D. }-0.08 \text {, } \\
\text { SE(m)- } 0.028\end{array}$ & \multicolumn{4}{|c|}{$\begin{array}{l}\text { C.D.- } 0.082, \\
\text { SE(m)-0.029 }\end{array}$} & $\begin{array}{l}\text { C.D. }-0.041 \text {, } \\
\text { SE(m)-0.014 }\end{array}$ \\
\hline Rootstock & 8.2 & 9.3 & 9.2 & 9.4 & 9.03 & 9.3 & 9.49 & 11.2 & 9.61 & 9.91 \\
\hline Mean & \multicolumn{5}{|c|}{ C.D. -0.071 SE $(\mathrm{m})-0.025$} & \multicolumn{5}{|c|}{ C.D. $-0.037 . \mathrm{SE}(\mathrm{m})-0.013$} \\
\hline
\end{tabular}

Cleopatra tangerine (TC); Rangpur Lime (RL); Swingle citrumelo (CS); Volkameriana (Vol); C.D. = coefficient desviation and SE - error standard.

Table 3. Scion circumference $(\mathrm{cm})$ of five sweet orange cultivars on four rootstocks (Maharashtra State, India)

\begin{tabular}{|c|c|c|c|c|c|c|c|c|c|c|}
\hline \multirow[b]{2}{*}{$\begin{array}{c}\text { Variety/ } \\
\text { Rootstock }\end{array}$} & \multicolumn{5}{|c|}{ Scion circumference $(\mathrm{cm}) 4^{\text {th }}$ year } & \multicolumn{5}{|c|}{ Scion circumference $(\mathrm{cm}) 4^{\text {th }}$ year } \\
\hline & $\mathrm{TC}$ & RL & CS & Vol & $\begin{array}{l}\text { Varietal } \\
\text { Mean }\end{array}$ & $\mathrm{TC}$ & RL & $\mathrm{CS}$ & Vol & $\begin{array}{l}\text { Varietal } \\
\text { Mean }\end{array}$ \\
\hline Hamlin & 23 & 22.3 & 24.5 & 25.8 & 23.89 & 25.7 & 26.1 & 26.8 & 29.3 & 26.98 \\
\hline Natal & 20.4 & 23 & 24.8 & 21.7 & 22.48 & 22.4 & 25.4 & 26.6 & 24.2 & 24.65 \\
\hline Pera & 19.9 & 21.7 & 22.9 & 23.1 & 21.88 & 22.7 & 23.8 & 26.5 & 24.8 & 24.23 \\
\hline Valencia & 26.1 & 26.4 & 23.3 & 22.3 & 24.53 & 27.4 & 27.4 & 26.1 & 24.1 & 26.23 \\
\hline Westin & 21.6 & 22.4 & 16.2 & 23.3 & 20.89 & 22.5 & 24.5 & 19.3 & 24.5 & 22.73 \\
\hline $\begin{array}{c}\text { Interaction } \\
\text { Mean }\end{array}$ & \multicolumn{4}{|c|}{$\begin{array}{l}\text { C.D.-1.785, } \\
\text { SE(m)-0.621 }\end{array}$} & $\begin{array}{l}\text { C.D.-0.893, } \\
\text { SE(m)-0.311 }\end{array}$ & \multicolumn{4}{|c|}{$\begin{array}{l}\text { C.D.-1.516, } \\
\text { SE(m)-0.528 }\end{array}$} & $\begin{array}{l}\text { C.D.- } 0.758, \\
\text { SE(m)-0.264 }\end{array}$ \\
\hline Rootstock & 22.2 & 23.2 & 22.3 & 23.3 & & 24.1 & 25.5 & 24.9 & 25.4 & \\
\hline Mean & \multicolumn{5}{|c|}{ C.D. $-0.798, \mathrm{SE}(\mathrm{m})-0.278$} & \multicolumn{5}{|c|}{ C.D.- $0.678, \mathrm{SE}(\mathrm{m})-0.236$} \\
\hline
\end{tabular}

Cleopatra tangerine (TC); Rangpur Lime (RL); Swingle citrumelo (CS); Volkameriana (Vol); C.D. = coefficient desviation and SE - error standard. 
influence by rootstocks. In a similar study on high density of sweet orange Wheaton et al. (1990) reported that all five varieties and rootstock combinations produced good quality fruits with only minor and inconsistent effects of stock on fruit weight.

\section{Juice content}

In fifth year age of plants, highest juice percentage was recorded in fruits harvested from Pera and Natal followed by Hamlin (Table 5). Juice content from both these cultivars was statistically at par but significantly higher than from rest of varieties under investigation. Hamlin produced higher juice content in previous studies (Chahal \& Gill, 2015). Minimum juice percentage was recorded from fruits of Westin cultivar. Non-significant variation in juice content was observed between all the rootstock in the present investigation however Wheaton et al. (1991) reported $51 \%$ to $60 \%$ juice content on different rootstocks with highest in fruit from trees on Swingle rootstock.

\section{Total soluble solids}

Fifth year age of plants, presented in Table 6 regarding Total Soluble Solids (TSS) show that fruits of Westin and Hamlin cultivars recorded maximum level of this parameters followed by remaining three cultivars showed statistically at par. Among the four rootstocks, plants on Cleopatra tangerine resulted into maximum TSS content in fruit juice and were significantly higher than those budded on remaining three rootstocks. However maximum Brix level was produced by fruits of Hamlin on Cleopatra tangerine combination and it was significantly higher than all other combinations. These results are in accordance

Table 4. Fruit weight (g) of five sweet orange cultivars on four rootstocks (Maharashtra State, India)

\begin{tabular}{|c|c|c|c|c|c|c|c|c|c|c|}
\hline \multirow[b]{2}{*}{$\begin{array}{l}\text { Variety/ } \\
\text { Rootstock }\end{array}$} & \multicolumn{5}{|c|}{ Fruit weight $(\mathrm{g}) 4^{\text {th }}$ year } & \multicolumn{5}{|c|}{ Fruit weight $(\mathrm{g}) 4^{\text {th }}$ year } \\
\hline & $\mathrm{TC}$ & RL & CS & Vol & $\begin{array}{l}\text { Varietal } \\
\text { Mean }\end{array}$ & $\mathrm{TC}$ & RL & $\mathrm{CS}$ & Vol & $\begin{array}{l}\text { Varietal } \\
\text { Mean }\end{array}$ \\
\hline Hamlin & 124.8 & 127.3 & 123.2 & 129.7 & 128.52 & 122 & 127 & 133 & 125 & 126.75 \\
\hline Natal & 184.9 & 191.3 & 224.1 & 187.7 & 196.99 & 182 & 193 & 225 & 185 & 196.25 \\
\hline Pera & 165.6 & 161.7 & 183.5 & 161.7 & 168.12 & 153 & 160 & 178 & 165 & 164 \\
\hline Valencia & 201 & 193.6 & 190.9 & 153.7 & 184.78 & 201 & 175 & 185 & 150 & 177.75 \\
\hline Westin & 128.2 & 189.2 & 164.4 & 195.9 & 169.43 & 128 & 189 & 169 & 196 & 170.5 \\
\hline $\begin{array}{l}\text { Interaction } \\
\text { Mean }\end{array}$ & \multicolumn{4}{|c|}{$\begin{array}{l}\text { C.D.-17.68, } \\
\text { SE(m)-6.154 }\end{array}$} & $\begin{array}{l}\text { C.D.-8.843, } \\
\text { SE(m)-3.077 }\end{array}$ & \multicolumn{4}{|c|}{$\begin{array}{l}\text { C.D. }-17.671 \text {, } \\
\text { SE(m)-6.783 }\end{array}$} & $\begin{array}{l}\text { C.D.-8.543, } \\
\text { SE(m)-2.677 }\end{array}$ \\
\hline Rootstock & 160.9 & 172.6 & 179.3 & 165.7 & & 178 & 169 & 178 & 164 & 167.05 \\
\hline Mean & \multicolumn{5}{|c|}{ C.D.-7.91,SE(m)-2.752 } & \multicolumn{5}{|c|}{ C.D.-6.149,SE(m)-2.571 } \\
\hline
\end{tabular}

Cleopatra tangerine (TC); Rangpur Lime (RL); Swingle citrumelo (CS); Volkameriana (Vol); C.D. = coefficient desviation and SE - error standard.

Table 5. Juice content (\%) of five sweet orange cultivars on four rootstocks (Maharashtra State, India)

\begin{tabular}{|c|c|c|c|c|c|}
\hline \multirow{2}{*}{ Variety/ Rootstock } & \multicolumn{5}{|c|}{ Juice content $(\%) 5^{\text {th }}$ year } \\
\hline & $\mathrm{TC}$ & RL & $\mathrm{CS}$ & Vol & Varietal Mean \\
\hline Hamlin & 48.4 & 48.3 & 44 & 50.3 & 47.75 \\
\hline Natal & 55.2 & 49.6 & 43.7 & 55.5 & 50.99 \\
\hline Pera & 56.1 & 55.3 & 44.5 & 48.6 & 51.11 \\
\hline Valencia & 42.9 & 54 & 47 & 42.5 & 46.6 \\
\hline Westin & 42.6 & 50 & 48.3 & 43 & 45.94 \\
\hline Interaction Mean & \multicolumn{2}{|c|}{$\begin{array}{l}\text { C.D.-3.301, } \\
\text { SE(m)-1.149 }\end{array}$} & & \multicolumn{2}{|c|}{$\begin{array}{l}\text { C.D.-2.984, } \\
\text { SE(m)-1.534 }\end{array}$} \\
\hline Rootstock Mean & 49 & 51.4 & $\begin{array}{r}45.5 \\
78, \mathrm{SE}(\end{array}$ & 48 & 48.47 \\
\hline
\end{tabular}

Cleopatra tangerine (TC); Rangpur Lime (RL); Swingle citrumelo (CS); Volkameriana (Vol); C.D. = coefficient desviation and SE - error standard. 
Table 6. Total Soluble Solids (TSS, \%) of five sweet orange cultivars on four rootstocks (Maharashtra State, India)

\begin{tabular}{|c|c|c|c|c|c|}
\hline \multirow{2}{*}{ Variety/ Rootstock } & \multicolumn{5}{|c|}{ TSS $(\%) 5$ th year } \\
\hline & $\mathrm{TC}$ & $\mathrm{RL}$ & $\mathrm{CS}$ & Vol & Varietal Mean \\
\hline Hamlin & 9.6 & 8.5 & 9.0 & 7.8 & 8.68 \\
\hline Natal & 7.5 & 8.5 & 7.0 & 7.8 & 7.75 \\
\hline Pera & 8.4 & 8.1 & 8.0 & 7.7 & 8 \\
\hline Valencia & 8.2 & 7.7 & 8.0 & 7.4 & 7.7 \\
\hline Westin & 8.9 & 7.9 & 9.0 & 8.8 & 8.7 \\
\hline Interaction Mean & \multicolumn{4}{|c|}{$\begin{array}{l}\text { C.D.- } 0.43 \text {, } \\
\text { SE(m)-0.15 }\end{array}$} & $\begin{array}{l}\text { C.D.-0.68, } \\
\text { SE(m)-0.14 }\end{array}$ \\
\hline Rootstock Mean & 8.5 & 8.1 & $\begin{array}{r}8.0 \\
4, \mathrm{SE} \\
\end{array}$ & C.D. $-0.34, \operatorname{SE}(\mathrm{m})-0.12$ & 8.17 \\
\hline
\end{tabular}

Cleopatra tangerine (TC); Rangpur Lime (RL); Swingle citrumelo (CS); Volkameriana (Vol); C.D. = coefficient desviation and SE - error standard.

Table 7. Yield $\left(\mathrm{kg} \mathrm{plant}^{-1}\right)$ and $\left(\mathrm{t} \mathrm{ha}^{-1}\right)$. of five sweet orange cultivars on four rootstocks (Maharashtra State, India)

\begin{tabular}{|c|c|c|c|c|c|c|c|c|c|c|}
\hline \multirow{2}{*}{$\begin{array}{c}\text { Variety/ } \\
\text { Rootstock }\end{array}$} & \multicolumn{5}{|c|}{ Yield $\left(\mathrm{kg} \mathrm{plant}^{-1}\right) 5^{\text {th }}$ year } & \multicolumn{5}{|c|}{ Yield $\left(\mathrm{t} \mathrm{ha}^{-1}\right) 5^{\text {th }}$ year } \\
\hline & TC & $\mathrm{RL}$ & $\mathrm{CS}$ & Vol & Varietal Mean & $\mathrm{TC}$ & RL & $\mathrm{CS}$ & Vol & Varietal Mean \\
\hline Hamlin & 41.1 & 26.4 & 62.6 & 30 & 40.03 & 34.2 & 22.0 & 52.1 & 25.0 & 33.3 \\
\hline Natal & 39.6 & 43.8 & 67.1 & 33 & 45.88 & 32.9 & 36.4 & 55.8 & 27.5 & 38.2 \\
\hline Pera & 22.2 & 25.6 & 32.3 & 26.9 & 26.75 & 18.5 & 21.3 & 26.9 & 22.4 & 22.3 \\
\hline Valencia & 40.3 & 26.3 & 59.5 & 11.3 & 34.35 & 33.5 & 21.9 & 49.5 & 9.4 & 28.6 \\
\hline Westin & 31 & 38.4 & 24 & 36.4 & 32.45 & 25.8 & 31.9 & 20.0 & 30.3 & 27.0 \\
\hline $\begin{array}{c}\text { Interaction } \\
\text { Mean }\end{array}$ & C.I & -9.66 & $\mathrm{E}(\mathrm{m})$ & 3.36 & $\begin{array}{c}\text { C.D. }-56, \mathrm{SE}(\mathrm{m}) \\
-4.52\end{array}$ & \multicolumn{4}{|c|}{ C.D. $-9.66, \operatorname{SE}(\mathrm{m})-3.36$} & $\begin{array}{c}\text { C.D.- } \\
\text { 8.56,SE(m)-4.52 }\end{array}$ \\
\hline Rootstock & 34.84 & 32.1 & 49.1 & 27.52 & & 29.0 & 26.7 & 40.9 & 22.9 & \\
\hline Mean & \multicolumn{5}{|c|}{ C.D.-8.654,SE(m)-2.784 } & \multicolumn{5}{|c|}{ C.D.-8.654,SE(m)-2.784 } \\
\hline
\end{tabular}

Cleopatra tangerine (TC); Rangpur Lime (RL); Swingle citrumelo (CS); Volkameriana (Vol); C.D. = coefficient desviation and SE - error standard.

with previous report by Chahal \& Gill (2015) where they reported significant variation in TSS level of different sweet orange varieties; cultivar budded on Volkameriana rootstock recorded minimum TSS level.

\section{Yield efficiency}

During $5^{\text {th }}$ year of plantation Natal recorded highest yield per plant followed by Hamlin (Table 7). All these three varieties however showed non-significant variation between each other. Various rootstocks had significant effect on yield per plant. Influence of rootstock-scion combinations on yield per plant revealed that plants of Natal budded on Swingle citrumelo resulted into maximum yield per plant. However lowest yield was observed in Valencia budded on Volkameriana. We could categorize yield in four group: very good (>50), good (35-50), average (20-35) and poor $(<20)$. Hamlin, Natal and Valencia on Swingle citrumelo can be grouped very good yielder, Hamlin, Natal and Valencia on Cleopatra tangerine, Natal and Westin on Volkameriana can be categorized as good yield. Rest of the combination was in average yielding category except Volkameriana that had a poor yield. Wheaton et al. (1991) studied cumulative yield, four cultivar for first 4 year of production varied considerably among scion and stock combination ranging from $46 \mathrm{t}$ ha to $233 \mathrm{t} / \mathrm{ha}$, Swingle and Sour orange rootstocks performed well with most scion cultivars.

Recently Chahal \& Gill (2015) recorded highest yield efficiency with Hamlin variety.

\section{ACKNOWLEDGEMENTS}

The authors are thankful to Jain Irrigation Systems Ltd., Jalgaon for financial support and permission. 


\section{REFERENCES}

Chahal TS \& Gill PPS (2015) Performance of Exotic Sweet Orange (Citrus sinensis Osbeck) Cultivars on Different Rootstocks under North Western India. Indian Journal of Science and Technology 8(16): 59391.

NHB - National Horticulture Board (2015). Indian horticulture databases. Gurgaon: National Horticulture Board. 282 p.
Wheaton TA, Castle WS, Whitney JD, Tucker DPH \& Muraro RP (1990) A high density citrus planting. Proceedings of the Annual Meeting of the Florida State Horticultural Society 103: 55-59.

Wheaton TA, Castle WS, Whitney JD \& Tucker DPH (1991) Performance of citrus scion cultivars and rootstock in high -density planting. HortScience 26(7): 837-840.

Received: March 30, 2017 Accepted: November 01, 2017 